\title{
Velocidad de crecimiento de grietas por corrosión bajo tensión en
} soldaduras $\operatorname{disímiles}^{(\cdot)}$

\author{
María del Pilar Fernández*, Jesús Lapeña*, Ana María Lancha*, \\ Francisco Javier Perosanz y Marta Navas*
}

Resumen

\begin{abstract}
Las soldaduras disímiles, que son utilizadas para unir diferentes partes en los reactores de agua ligera, son potencialmente susceptibles de experimentar corrosión bajo tensión (SCC) en los medios acuosos característicos de las plantas nucleares. Sin embargo, el estudio de estas soldaduras ha estado limitado a evaluar en dichos medios la susceptibilidad a SCC de los materiales que componen las mismas por separado, existiendo pocos datos de velocidad de crecimiento de grietas debido fundamentalmente a la falta de estandarización de este tipo de ensayos. En este trabajo se ha pretendido determinar la velocidad de crecimiento de grietas en la interfase formada por el acero ferrítico SA533B-1 y la aleación I-82 dentro de la soldadura disímil compuesta por el acero SA533B-1/I-82/316L en unas condiciones de química del agua correspondientes a $8 \mathrm{ppm}$ de $\mathrm{O}_{2}$, una conductividad de $1 \mathrm{fS} / \mathrm{cm}$ y a una temperatura de $288^{\circ} \mathrm{C}$.
\end{abstract}

Palabras clave Soldaduras disímiles, Corrosión bajo tensión. Velocidad de crecimiento de grietas. Corrosión en agua.

\section{Stress corrosion crack growth rate in dissimilar metal welds}

\begin{abstract}
Dissimilar welds, used to join different sections in light water reactors, are potentially susceptible to stress corrosion cracking (SCC) in aqueous mediums characteristic of nuclear plants. However, the study of these welds has been limited to evaluating the weld material susceptibility in these mediums. Little scarce data are available on crack growth rates due, fundamentally, to inadequate testing techniques. In order to address this lack of information the crack growth rate at the interface of ferritic SA533B-1 alloy and alloy I-82, in a dissimilar weld (SA533B-1/I-82/316L), was studied. Experiments were conducted in water at $288^{\circ} \mathrm{C}, 8 \mathrm{ppm}$ of $\mathrm{O}_{2}$ and $1 \mu \mathrm{S} / \mathrm{cm}$ conductivity.
\end{abstract}

Keywords Dissimilar metal welds. Stress corrosion cracking. Crack growth rate. Water corrosion.

\section{INTRODUCCIÓN}

Las soldaduras de metales disímiles (DMWs) se usan en una gran variedad de componentes en los reactores de agua ligera para unir aceros ferríticos de baja aleación a aceros inoxidables austeníticos, o aceros de baja aleación a aleaciones base níquel.

Estas soldaduras disímiles se utilizan ampliamente en diferentes partes de una planta nuclear como son las conexiones de las tuberías a la vasija del reactor. En estas conexiones, las tuberías son, usualmente, de aceros inoxidables austeníticos, mientras que la vasija está fabricada con acero ferrítico de baja aleación, por lo que, generalmente, se emplea para su unión un material de soldadura compatible con los materiales que se van a soldar. Este tipo de uniones también se realizan en los presionadores, en la carcasa de los generadores de vapor y en las penetraciones de la vasija. La utilización de este tipo de soldaduras no es exclusivo de las plantas nucleares, sino que también se emplean en sistemas de conversión de energía a alta temperatura $^{[1]}$.

Independientemente de cual sea el material de soldadura, el proceso de soldadura empleado y el tratamiento térmico posterior, pueden producirse algunos cambios microestructurales en la interfase, como por ejemplo, pequeños precipitados,

(•) Trabajo recibido el día 12 de febrero de 1999 y aceptado en su forma final el 13 de enero de 2000.

$\left(^{*}\right)$ Proyecto de Materiales Estructurales en Plantas Energéticas, DFN, CIEMAT. Avda. Complutense, 22. 28040 Madrid (España). 
aumento de tamaño de grano, etc, que en consecuencia pueden originar cambios en sus propiedades metalúrgicas. Estas modificaciones de sus características metalúrgicas pueden hacer que estos materiales, en determinados ambientes, sean potencialmente susceptibles de experimentar fenómenos de degradación por corrosión localizada ${ }^{[2-4]}$ y, en particular, agrietamiento asistido por el ambiente (Environmentally Assisted Cracking, EAC), a través de una gran variedad de mecanismos. Todos estos procesos de corrosión están en función de los materiales que componen la soldadura, de sus características metalúrgicas y del medio al que están expuesto ${ }^{[2-6]}$.

En la mayoria de los casos, el trabajo experimental realizado en este tipo de soldaduras ha estado generalmente limitado a evaluar la susceptibilidad de las aleaciones que componen la soldadura en los medios acuosos característicos del funcionamiento de los reactores nucleares. Sin embargo, el conocimiento y entendimiento de los mecanismos de corrosión bajo tensión (SCC) en estos materiales es limitado, factor que puede ser debido, fundamentalmente, a la gran complejidad de los mecanismos de rotura y, quizás, a que las técnicas de ensayo empleadas no han sido bien desarrolladas o estandarizadas. Por todos estos factores, existe la necesidad de desarrollar métodos apropiados de ensayo bajo los cuales se produzca el agrietamiento para poder determinar su localización en la soldadura y, además, generar datos de la velocidad de crecimiento de las grietas. Con el objetivo de resolver toda esta serie de necesidades surge dentro de la Comunidad Económica Europea el proyecto denominado Evaluation of Techniques for Assessing Corrosion Cracking in Dissimilar Metal Welds (DISWEC), en el que han formado parte ocho laboratorios incluido el Proyecto de Materiales Estructurales del CIEMAT.

El objetivo y alcance del proyecto DISWEC ha consistido en el estudio de la susceptibilidad y la obtención de datos cuantitativos de velocidad de crecimiento de grietas en una soldadura disímil, especialmente preparada para este proyecto, formada por el acero ferrítico SA533B-1/ Inconel 82/ acero inoxidable $316 \mathrm{~L}$, representativa de las modernas soldaduras disímiles existentes en la industria nuclear.

Para cumplir con los objetivos del proyecto se diseñó un trabajo experimental basado fundamentamente en dos partes:

- Estudio comparativo de las diferentes técnicas de ensayo que se pueden utilizar para la evalua- ción de la susceptibilidad a experimentar la corrosión bajo tensión en los medios de operación de los reactores de agua ligera.

- Evaluación de la compatibilidad de diferentes métodos de ensayo para la determinación de la velocidad de crecimiento de grietas en las regiones más susceptibles de experimentar corrosión bajo tensión.

El trabajo desarrollado en el CIEMAT, se ha centrado en el estudio de la velocidad de crecimiento de grietas bajo carga constante en la interfase formada por el acero SA533B-1/ I-82 de la soldadura disímil objeto de estudio (SA533B-1/ I-82/AISI 316L), de modo que los datos obtenidos sirvan para el análisis de defectos en las plantas nucleares.

Revisiones bibliograficas efectuadas por todos los laboratorios integrantes del proyecto, con el objetivo de poder comparar resultados y optimizar los métodos de ensayo, mostraron que desafortunadamente no existen trabajos publicados en los que se recojan estudios realizados en soldaduras disímiles formadas exactamente por dichos materiales. Algunos de los resultados publicados más relevantes ${ }^{[7-18]}$ son datos obtenidos en las soldaduras disímiles formadas por el acero A508/ I-82, I-82/ AISI 316 , I-82/AISI $316 \mathrm{NG}$ e I-82 junto con otros aceros inoxidables o aleaciones base níquel.

\section{INSTALACIÓN Y EQUIPOS}

Todos los ensayos de velocidad de crecimiento de grietas se han realizado en una instalación que consta de un sistema de purificación y acondicionamiento del agua y de un sistema de alta presión y temperatura. El primer sistema mencionado permite obtener agua de calidad reactor y simular los distintos tipos de química que sean requeridos para cada tipo de ensayo. Para ello, en una primera fase se purifica el agua a través de una serie de filtros entre los que se incluye un filtro de carbón activo y un equipo de ósmosis inversa. Posteriormente, pasa a través de un equipo super $\mathrm{Q}$, compuesto por dos resinas de ácidos y bases fuertes, un cartucho de carbón activo y un filtro de membrana de 0,22 $\mu \mathrm{m}$ de tamaño de poro, llegándose a obtener agua de $0,056 \mu \mathrm{S} / \mathrm{cm}$ de conductividad, con un caudal de producción de $100 \mathrm{~L} / \mathrm{h}$, notablemente superior al previsto en el ensayo, por lo que se almacena en un depósito, estableciéndose un funcionamiento intermitente de acuerdo con las necesidades. Una vez purificada, ésta se acondiciona en los tanques 
de alimentación según la química necesaria para el ensayo.

El acondicionamiento se realiza por medio del burbujeo de una mezcla de gases, oxígeno-argón, lo que permite obtener las concentraciones de oxígeno requeridas en cada ensayo.

Durante los ensayos se mide el oxígeno y la conductividad a la entrada y salida de los circuitos, utilizándose para ello analizadores de oxígeno Beckman modelo 701 y 735X, y conductímetros Kent-5217. Periódicamente, se controla la concentración de especies disueltas a la entrada y salida del circuito. El análisis químico de los aniones se lleva a cabo por cromatografía iónica, y el de los cationes por espectroscopía de emisión atómica de plasma.

Una vez que se ha obtenido el agua purificada y acondicionada, se procede a su calentamiento hasta alcanzar la temperatura de trabajo que, en este caso, es de $288^{\circ} \mathrm{C}$ y una presión de $80 \mathrm{~kg} / \mathrm{cm}^{2}$ en el sistema de alta presión y temperatura.

Todos estos circuitos están conectados a una autoclave de acero inoxidable AISI 316 de $8 \mathrm{~L}$ de capacidad, que a su vez está acoplada a una máquina de ensayos mecánicos MTS 810 de 10 t, en la que se realizan los ensayos de velocidad de crecimiento de grietas con probetas $\mathrm{CT}^{[19]}$, con la posibilidad de poder ensayar dos probetas simultáneamente. Para la medida de los potenciales electroquímicos se dispone de una autoclave de $1 \mathrm{~L}$ de capacidad, que se encuentra situada aguas abajo de la autoclave de los ensayos CT. Teniendo en cuenta que el recorrido entre dichas autoclaves es corto, es válido considerar que las condiciones químicas son las mismas que en la autoclave de trabajo.

El medio seleccionado en principio correspondía a las condiciones de operación de los reactores de agua en ebullición (BWR), que trabajan con concentraciones de $\mathrm{O}_{2}$ tanto en química normal (NWC) como en química alternativa (HWC), del orden de 200 y $20 \mathrm{ppb}$ respectivamente, y una conductividad $<0,3 \mu \mathrm{S} / \mathrm{cm}$, a temperatura de 288 ${ }^{\circ} \mathrm{C}$. No obstante, con el fin de obtener valores conservativos, el medio elegido para los ensayos intenta reproducir las condiciones más desfavorables que se presentan en planta, como son las paradas, durante las cuales se pueden alcanzar concentraciones de $\mathrm{O}_{2}$ del orden de $8 \mathrm{ppm} \mathrm{y}$ conductividades elevadas $(1 \mu \mathrm{S} / \mathrm{cm})$. Para la obtención de una conductividad de $1 \mu \mathrm{S} / \mathrm{cm}$ se añadieron sulfatos en una concentración de $105 \mathrm{ppb}$., en forma de sulfúrico.
Para la medida de los potenciales electroquímicos de corrosión se emplearon como electrodos de referencia $\mathrm{Ag} / \mathrm{AgCl}$ y un electrodo cerámico de $\mathrm{Cu} / \mathrm{Cu}_{2} \mathrm{O}^{[20]}$. Sin embargo, debido a la alta concentración de $\mathrm{O}_{2}$ se detectó un funcionamiento anómalo del electrodo de plata por lo que se retiró en las últimas medidas. Los electrodos de trabajo se fabricaron a partir de los materiales que forman la unión a estudiar, es decir uno de acero ferrítico SA533B-1 y el otro de I-82 depositado.

Todas las variables involucradas en el ensayo (presión, temperatura, conductividad, concentración de $\mathrm{O}_{2}$, potenciales de corrosión), así como medidas de la longitud de la grieta, mediante técnicas de caída de potencial ${ }^{[21]}$, fueron monitorizadas en contínuo mediante un software de desarrollo propio basado en conceptos de instrumentación virtual. Con este sistema, además de recoger y almacenar las señales y parámetros descritos es posible corregir inmediatamente cualquier anomalía detectada.

Las medidas de longitud de grieta mediante la técnica de caída de potencial ${ }^{[21]}$ se realizan haciendo pasar una corriente continua o alterna a través del material objeto de estudio. Cualquier crecimiento de la grieta se registra como una variación de la diferencia de potencial establecida entre dos puntos de la probeta. El equipo de caída de potencial utilizado para los ensayos se ha desarrollado en el CIEMAT, utilizándose corriente alterna de excitación de forma sinusoidal de $20 \mathrm{~Hz}$. Este método únicamente se empleó en dos de los ensayos realizados.

\section{MATERIALES}

Como se ha mencionado con anterioridad, los materiales que componen la soldadura disímil son los del acero ferrítico SA533B-1/I-82/ AISI 316L.

El proceso de soldadura de estos materiales se efectuó en dos secuencias. Primeramente, mediante soldadura TIG, se depositó sobre el acero SA533B-1 una gruesa capa de la aleación base níquel I-82, empleando esta aleación como material de aporte. Posteriormente, se soldó la capa mencionada con el acero inoxidable austenítico 316L utilizando la misma técnica y material de aporte.

Mediante diversos ensayos realizados por los demás participantes en el mencionado proyecto, se determinó que la interfase formada por el acero ferrítico SA533B-1 y el I-82 era la más susceptible a experimentar EAC, por lo que el estudio experimental se ha centrado en la determinación de 
velocidad de crecimiento de grietas en la interfase formada por estos dos materiales cuya composición química queda reflejada en la tabla I.

Para la obtención de estos datos de la velocidad de crecimiento de grietas se mecanizaron siete probetas CT de 1 pulg. de espesor a partir de la soldadura, de acuerdo con la norma ASTM E399 ${ }^{[19]}$, según puede verse en la figura 1 , situando la entalla en la interfase formada por el acero SA533B-1 y la capa añadida del material I-82.

En el fondo de la entalla mecánica se provocó antes del ensayo una grieta de fatiga en aire a temperatura ambiente, para favorecer su posterior propagación durante el ensayo de corrosión bajo tensión. Para generar la grieta de fatiga, se sometió la probeta a carga cíclica, con ciclos en forma de diente de sierra. El factor de intensidad de tensiones máximo $(K)$ durante este preagrietamiento fue siempre inferior al del período de propagación.

\subsection{Caracterización microestructural de la sol- dadura}

Previamente a la realización de los ensayos de velocidad de crecimiento de grietas, se efectuó la caracterización microestructural por el conjunto de laboratorios integrantes del proyecto. Los aspectos microestructurales más destacables de la interfase de soldadura (SA533B-1/I-82) se detallan a continuación.

Se obtuvieron valores de microdureza (HV200) de hasta 450 en ciertas áreas del material I-82 muy próximas a la interfase, superiores a la media en ese área que fue de 250. Mediante microscopía

Tabla I. Composición química de los materiales, \% en peso

Table I. Chemical composition of the materials, \% weight

\begin{tabular}{ccc}
\hline Elemento & $\mathrm{I}-82$ & SA533B-1 \\
\hline $\mathrm{C}$ & 0,036 & 0,215 \\
$\mathrm{~S}$ & 0,001 & 0,003 \\
$\mathrm{P}$ & 0,004 & 0,010 \\
$\mathrm{Si}$ & 0,05 & 0,220 \\
$\mathrm{Mn}$ & 2,92 & 1,430 \\
$\mathrm{Ni}$ & 73 & 0,670 \\
$\mathrm{Cr}$ & 20,23 & - \\
$\mathrm{Mo}$ & - & 0,490 \\
$\mathrm{Fe}$ & 0,71 & \\
$\mathrm{Nb}$ & 2,48 & \\
$\mathrm{Ti}$ & 0,38 & \\
$\mathrm{Cu}$ & 0,03 & \\
$\mathrm{Co}$ & 0,04 & \\
\hline
\end{tabular}

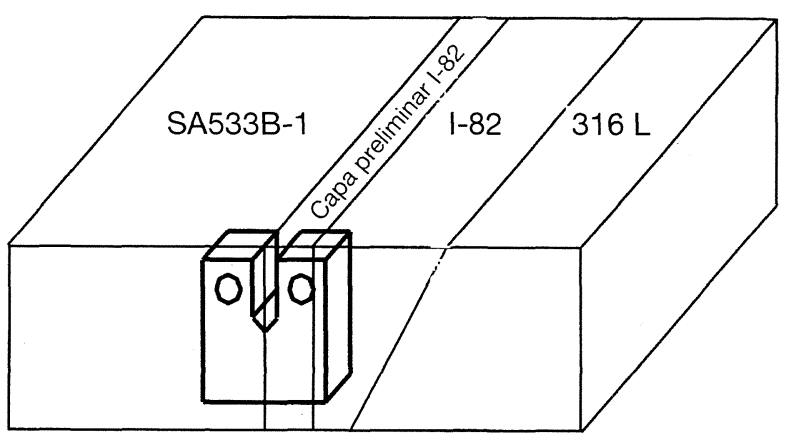

Figura 1. Mecanizado de las probetas a partir de la soldadura.

Figure 1. Specimens machined from the weld.

óptica, se observó que estos picos puntuales de dureza estaban asociados a la presencia de martensita sin transformar, que en algunas zonas se había formado a partir de la línea de fusión (Fig. 2). Mediante metalografía óptica también se midió el tamaño de grano del SA533B-1, presentando este material, en zonas adyacentes a la interfase, un tamaño de grano correspondiente a un valor medio de $4 \mu \mathrm{m}$. El estudio microestructural del I-82 únicamente reveló grandes granos columnares cerca de la interfase.

Mediante microscopía electrónica de barrido se detectaron dos tipos de inclusiones: inclusiones tipo A en el I-82 e inclusiones tipo B en el acero ferrítico. Para determinar de forma cualitativa la composición de estas inclusiones se utilizó la téc-nica de dispersión de energía de rayos $\mathrm{X}$ (EDX). Los análisis efectuados en las inclusiones tipo A dieron como resultado picos de energía

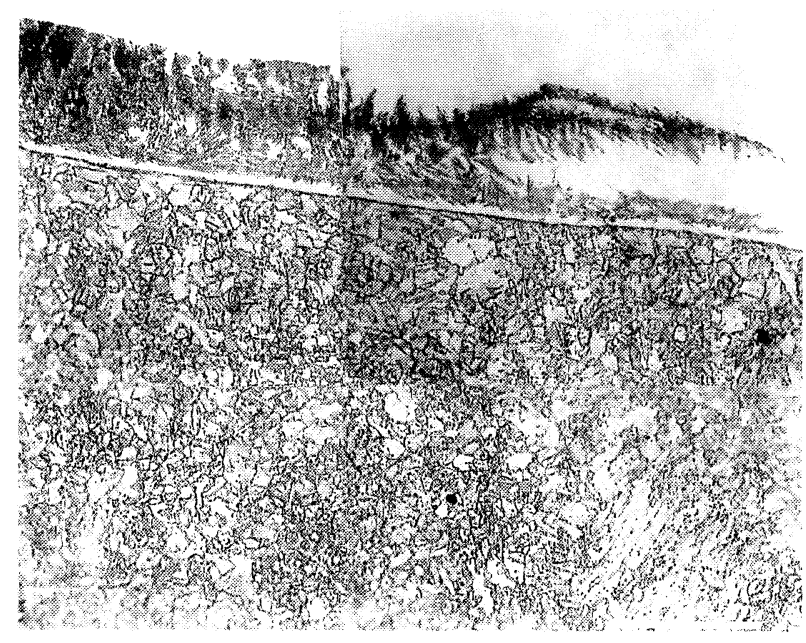

Figura 2. Detalle microestructural de la martensita formada cerca de la línea de fusión, en algunas zonas del material de soldadura.

Figure 2. Microstructural detail of martensite formed in some places of the weld material near the fusion line.

Rev. Metal. Madrid 36 (2000) 
correspondientes a oxígeno, aluminio y calcio. Debido a que estos elementos no forman parte de la composición del material de soldadura se piensa que fueron introducidos durante el proceso de soldadura. Por el contrario, las inclusiones observadas en el material base (SA533B-1) mostraron ser inclusiones de MnS. Utilizando esta misma técnica se caracterizó la distribución relativa de los elementos mayoritarios a través de la interfase (SA533B-1/I-82) observándose gradientes significativos de hierro y níquel. Concretamente, se vio que el hierro había difundido desde el acero ferrítico hacia el I-82 hasta una distancia de 4-5 mm y que su concentración era mayor a la esperada para esta aleación.

\section{RESULTADOS}

\subsection{Velocidad de crecimiento de grietas}

Las condiciones químicas de los ensayos de velocidad de crecimiento de grietas se indican en la tabla II. En la figura 3 se muestran las medidas de los potenciales de corrosión, durante uno de los ensayos (Probetas D2 y D8), en la que es posible observar un aumento del potencial de corrosión con el tiempo de ensayo debido a la oxidación de los electrodos de trabajo, como consecuencia del alto contenido de $\mathrm{O}_{2}$. En el resto de los ensayos, las medidas fueron similares, observándose un aumento del potencial de corrosión mayor cuando los electrodos se reemplazaban por otros electrodos nuevos. Teniendo en cuenta la gran dependencia del potencial electroquímico con el contenido de $\mathrm{O}_{2}$, el intervalo de los potenciales de corrosión está en concordancia con la alta concentración de estos ensayos.

De las siete probetas que se mecanizaron para los ensayos, solamente en dos (D5 y D8) se pudo situar el fondo de la entalla justamente en la interfase como se pretendía, debido a que la interfase de soldadura presentaba una superficie ondulada, lo que dio lugar a que cada probeta, dependiendo de la posición del fondo de la entalla (Interfase, SA533B-1, I-82) experimentara una localización particular de la pregrieta de fatiga y de la grieta de propagación,

Tabla II. Química del agua

Tabla II. Water chemistry

\begin{tabular}{cccc}
\hline $\begin{array}{c}\text { Conductividad } \\
\text { a la entrada }\end{array}$ & $\begin{array}{c}\text { Conductividad } \\
\text { a la salida }\end{array}$ & $\mathrm{SO}_{4}^{2-}$ & $\mathrm{O}_{2}$ (Salida) \\
\hline $0,9 \pm 0,15 \mu \mathrm{S} / \mathrm{cm}$ & $1,1 \pm 0,2 \mu \mathrm{S} / \mathrm{cm}$ & $105 \pm 15(\mathrm{ppb})$ & $8 \pm 1(\mathrm{ppm})$ \\
\hline
\end{tabular}

Rev. Metal. Madrid 36 (2000)

(c) Consejo Superior de Investigaciones Científicas

Licencia Creative Commons 3.0 España (by-nc)

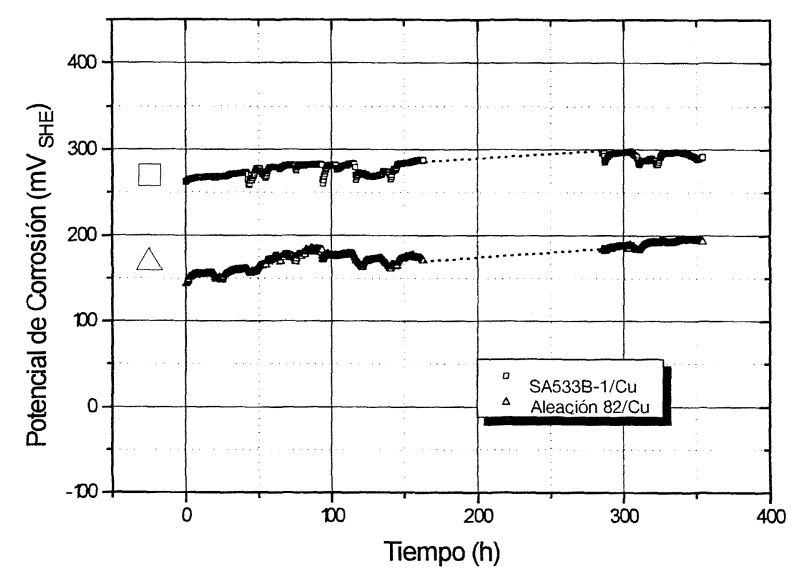

Figura 3. Potenciales de corrosión obtenidos en la probetas D2 y D8 reproducibles para los demás ensayos.

Figure 3: Corrosion potential for D2 and D8 specimens reproducible for the other tests.

como queda reflejado en la tabla III, en la que se resumen de forma esquemática las características mencionadas para cada una de las probetas.

Los ensayos de velocidad de propagación de grietas se efectuaron bajo carga constante, ensayándose dos probetas simultáneamente, excepto la D7 que fue ensayada sola. Esta probeta tiene la particularidad, con respecto a las demás, que fue ensayada a tres cargas diferentes, es decir, a partir de la carga inicial y transcurridas unas determinadas horas de ensayo se aumentó la carga en dos ocasiones. La carga aplicada en cada caso dependía de la longitud de la pregrieta formada por fatiga al aire y del factor de intensidad de tensiones $(K)$ que se pretendía obtener en cada ensayo, pero siempre dentro del intervalo de $K$ comprendido entre $35 \mathrm{y}$ $56 \mathrm{MPa} \sqrt{\mathrm{m}}$.

La técnica de caída de potencial para la medida de velocidad de crecimiento de grietas se utilizó únicamente en los ensayos de las probetas D4, D5 y D7.

El factor de intensidad de tensiones está relacionado con la carga proporcionada por la máquina de ensayo mediante la siguiente ecuación ${ }^{[19]}$ :

$$
\begin{gathered}
K=\frac{P}{B W^{1 / 2}} \frac{2+a / W}{(1-a / W)^{3 / 2}}\left[0,886+4,64(a / W)^{2}-\right. \\
\left.-13,32(a / W)^{2}+14,72(a / W)^{3}-5,6(a / W)^{4}\right]
\end{gathered}
$$

donde: $K=$ factor de intensidad de tensiones,

$P=$ carga,

$B=$ espesor de la probeta,

$W=$ distancia de la línea de actuación de carga hasta el fondo de la probeta,

$a=$ tamaño de la grieta. 
Velocidad de crecimiento de grietas por corrosión bajo tensión en...

Tabla III. Características de la localización de la grieta durante el ensayo en función de la posición de la entalla y de la grieta de fatiga

Table III. Characteristics of crack localization during the tests in function of notch and fatigue crack position

\begin{tabular}{cccc}
\hline No de probeta & Localización de la entalla & Localización de la grieta de fatiga & Localización de la grieta de corrosión bajo tensión \\
\hline D1 & SA533B-1 & SA533B-1 & SA533B-1 \\
D2 & SA533B-1 & SA533B-1 & SA533B-1, pero con tendencia hacia la interfase \\
D3 & $\begin{array}{c}\text { Cerca de la interfase } \\
\text { SA533B-1/I-82 }\end{array}$ & SA533B-1, cerca de la interfase & SA533B-1 \\
D4 & I-82 & I-82 & Interfase + unas zonas en el \\
& Interfase & SA533B-1 y otras zonas en I-82
\end{tabular}

Después del ensayo se rompieron las probetas por fatiga al aire y se midió el tamaño real de la grieta por microscopía estereoscópica. Las medidas se tomaron en nueve puntos equidistantes, calculando el valor medio resultante de la grieta por la siguiente ecuación ${ }^{[19]}$.

$$
\bar{a}=\frac{\frac{a_{1}+a_{9}}{2}+\sum_{i=2}^{8} a_{i}}{8}
$$

Los resultados numéricos obtenidos en los ensayos, junto con la duración de los mismos, se resumen en la tabla IV. La representación gráfica de la velocidad de crecimiento de grietas frente al factor de intensidad de tensiones $K_{\text {medio }}$, tomado como la media entre el $K_{\text {inicial }}$ al inicio del ensayo y el $K_{\text {final }}$ se encuentra en la figura 4.

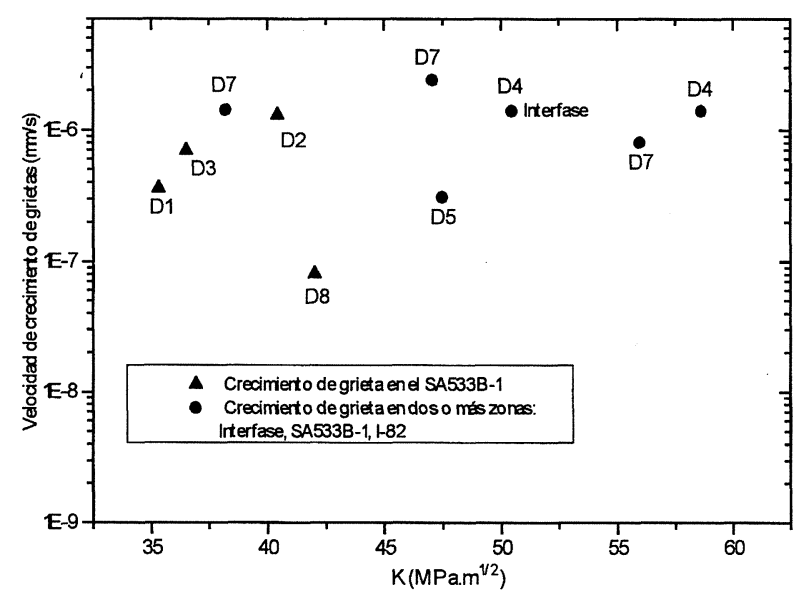

Figura 4. Velocidad de crecimiento de grietas frente al factor de intensidad de tensiones $(K)$.

Figure 4. Crack growth rate vs stress intensity factor (K).

Tabla IV. Resultados de los ensayos obtenidos bajo carga constante en probetas ITCT.

Table IV. Constant load test results on ITCT specimens

\begin{tabular}{|c|c|c|c|c|c|c|c|}
\hline & Horas de ensayo & $\begin{array}{c}a_{\mathrm{o}} \\
(\mathrm{mm})\end{array}$ & $\begin{array}{c}\Delta a \\
(\mathrm{~mm})\end{array}$ & $\begin{array}{c}d a / d t \\
(\mathrm{~mm} / \mathrm{s})\end{array}$ & $\frac{K_{\text {inicial }}}{(\mathrm{MPa} \sqrt{\mathrm{m})}}$ & $\frac{K_{\text {final }}}{(\mathrm{MPa} \sqrt{\mathrm{m})}}$ & $\frac{K_{\text {medio }}}{(\mathrm{MPa} \sqrt{\mathrm{m})}}$ \\
\hline D1 & 1.079 & 26,51 & 1,39 & $3,6 \cdot 10^{-7}$ & 33,7 & 37 & 35,3 \\
\hline D2 & 353 & 26,89 & 1,65 & $1,3 \cdot 10^{-6}$ & 38,2 & 42,6 & 40,4 \\
\hline D3 & 1.079 & 26,28 & 2,73 & $7,10^{-7}$ & 33,2 & 39,8 & 36,5 \\
\hline \multirow{2}{*}{ D4 } & 460 & 29,40 & 2,33 (Interfase) & $1,4 \cdot 10^{-6}$ & 45,9 & 55 & 50,4 \\
\hline & 284 & 31,73 & $1,41(1-82+S A 533 B-1)$ & $1,4 \cdot 10^{-6}$ & 55 & 62,3 & 58,6 \\
\hline \multirow[t]{2}{*}{ D5 } & 744 & 29,5 & 0,82 & $3,1 \cdot 10^{-7}$ & 46 & 48,9 & 47,5 \\
\hline & $1^{\text {a }}$ parte: 311 & $1^{\text {a }}$ parte: 27,5 & $1^{\text {a }}$ parte: 1,60 & $1^{\text {a }}$ parte: $1,4 \cdot 10^{-6}$ & $1^{\text {a }}$ parte: 36,1 & $1^{\text {a }}$ parte: 40,3 & $1^{\text {a }}$ parte: 38,2 \\
\hline \multirow[t]{2}{*}{ D7 } & $2^{a}$ parte: 152 & $2^{\text {a }}$ parte: 29,1 & $2^{\text {a }}$ parte: 1,33 & $2^{\text {a }}$ parte: $2,4 \cdot 10^{-6}$ & $2^{\mathrm{a}}$ parte: 44,7 & $2^{\text {a }}$ parte: 49,4 & $2^{\text {a }}$ parte: 47,1 \\
\hline & $3^{a}$ parte: 86 & $3^{a}$ parte: 30,7 & $3^{\text {a }}$ parte: 0,25 & $3^{a}$ parte: $8,1 \cdot 10^{-7}$ & $3^{\text {a }}$ parte: 55,4 & $3^{\text {a }}$ parte: 56,5 & $3^{\text {a }}$ parte: 56,0 \\
\hline D8 & 353 & 28,34 & 0,10 & $7,9 \cdot 10^{-8}$ & 42 & 42 & 42 \\
\hline
\end{tabular}




\subsection{Estudio fractográfico}

Mediante el estudio de las tablas III y IV puede observarse que en aquellas probetas (D1, D2 y D3) en las cuales la posición de la entalla y la pregrieta de fatiga están situadas en el acero ferrítico o muy cerca de la interfase, pero dentro del SA533B-1, el crecimiento se produjo en todas ellas de forma transgranular dentro del acero ferrítico. En la superficie de fractura, característica de este tipo de ensayos (Fig. 5), se pueden distinguir claramente la zona de la entalla, el área correspondiente al preagrietamiento por fatiga al aire, y la zona de propagación de la grieta, que en este caso ha tenido lugar de forma transgranular.

De estas tres probetas, cabe destacar la probeta D3, en la cual, durante el estudio de su superficie de fractura, se observó en el área de crecimiento por fatiga algunas zonas de material de soldadura (Fig. 6), identificadas en la macrografía con un 1. Este hecho puede ser debido a que la grieta de fatiga al aire se produjo muy cerca de la interfase y que la interfase de soldadura, como ya se ha mencionado, presenta una superficie ondulada.

En el mecanizado de las probetas D5 y D8 se posicionó el fondo de la entalla justamente en la interfase; sin embargo, el preagrietamiento se produjo en ambas dentro del acero ferrítico. La probeta D8, después de $353 \mathrm{~h}$ de ensayo, presentó un crecimiento insignificante de tipo transgranular en una pequeña área del acero SA533B-1. Por el contrario, la probeta $\mathrm{D} 5$, que no presentó crecimiento en sus superficies externas, una vez abierta para el examen de su superficie de fractura mostró que la grieta se había propagado en algunas zonas en el material de soldadura (I-82), de forma intergranular, y en otras áreas, en el acero SA533B-1, de forma transgranular. A priori, cabe pensar que este comportamiento se ha producido por la proximidad de la pregrieta a la línea de fusión; sin embar-

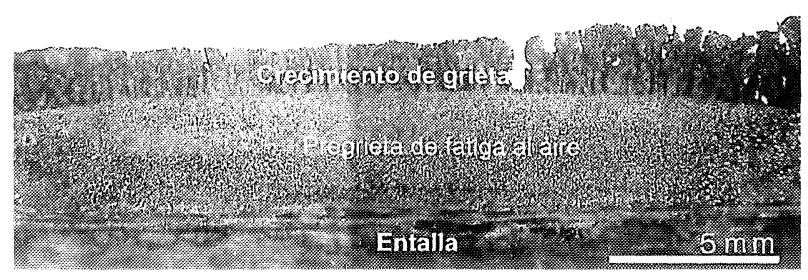

Figura 5. Superficie de fractura característica de los ensayos de velocidad de crecimiento de grietas bajo carga constante.

Figure 5. Characteristic fracture surface of crack growth rate tests under constant load.

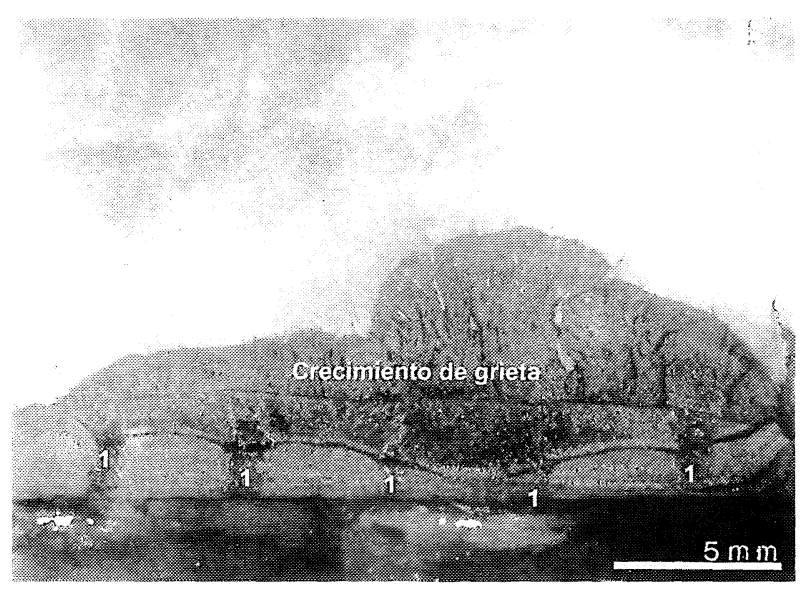

Figura 6. Superficie de fractura correspondiente a la probeta D3.

1) Zonas de material de soldadura.

Figure 6. Fracture surface of specimen D3.

1) Zones of weld material.

go, esta mezcla de zonas de crecimiento también se observó en las probetas D4 y D7, en las que tanto la entalla como la grieta de fatiga se localizaron en el material de soldadura (I-82) como se detalla a continuación.

En la probeta $\mathrm{D} 4$, primeramente hay que hacer una distinción entre las dos superficies externas de la probeta. En una de las caras (Cara A), la grieta de fatiga que comienza en el I-82 progresa hacia la interfase, produciéndose el crecimiento durante el ensayo, justamente en la línea de fusión. En la otra cara (Cara B), el preagrietamiento ocurre en el I-82, pero al comenzar el ensayo la grieta experimenta un salto hacia el acero ferrítico (SA533B1) progresando en este mismo material durante la mayor parte del ensayo hasta que al final la grieta propaga de nuevo en el I-82. El estudio de la superficie de fractura reveló que, al someter la probeta a carga, la grieta experimentó un salto en un plano diferente al plano del preagrietamiento, creciendo la grieta, primeramente, en la interfase SA533B-1/I-82 para pasar, posteriormente, a una fase de propagación en la que el avance de la misma tuvo lugar, en algunas partes, en el material de soldadura cerca de la interfase con un crecimiento intergranular $y$, en otras, se produjo de forma transgranular en el acero ferrítico (Fig. 7). Las zonas brillantes de la micrografía se corresponden con el crecimiento intergranular, mientras que las zonas oscuras representan el crecimiento en material ferrítico.

La probeta D7 se ensayó durante 549 h a tres cargas diferentes para obtener más información de la velocidad de crecimiento de grietas frente al 


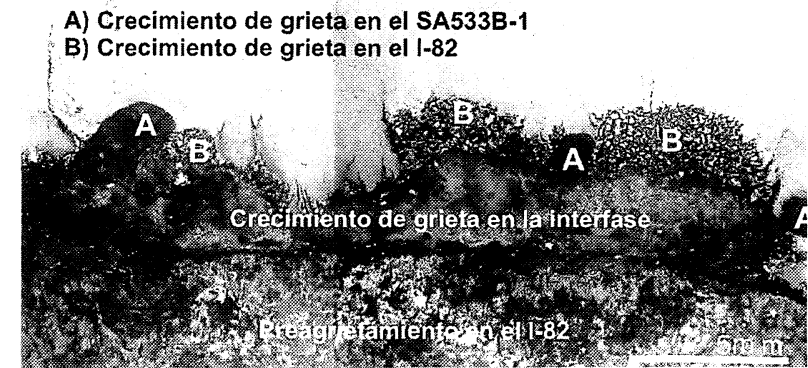

Figura 7. Superficie de fractura de la probeta D4.

Figure 7. Fracture surface of specimen D4.

factor de intensidad de tensiones. Al igual que la probeta D4, la entalla y la pregrieta estaban en el material de soldadura. La grieta, en una primera parte, crece en el material de soldadura, mientras que en una segunda fase experimenta un salto a partir del cual, en algunas zonas avanza en, o muy cerca, de la interfase y, en otras, el crecimiento tiene lugar en el acero ferrítico.

\section{DISCUSIÓN}

Se puede realizar un primer análisis considerando la zona donde se ha producido la propagación de grietas en cada probeta. Respecto a las cuatro que han propagado exclusivamente en el acero ferrítico, como puede verse en la tabla III, para poder evaluar los resultados hay que tener en consideración algunas características particulares de los ensayos realizados.

Primeramente, hay que considerar que el material ferrítico se encuentra unido a una aleación base níquel (I-82), y aunque el crecimiento se ha producido en dicho material, la grieta en algunas probetas se encontraba solamente a $0,1 \mathrm{~mm}$ de la línea de fusión. Además, las probetas han sido sometidas a carga constante, pero en la mayoría de los casos se han producido pequeñas interrupciones por diferentes causas durante las cuales las probetas estuvieron descargadas. Por último, hay que considerar la influencia de la concentración de azufre en el frente de la grieta. Este azufre es el resultante del equilibrio dinámico que se establece entre el proveniente de la disolución de las inclusiones de $\mathrm{MnS}$ y el de los sulfatos contenidos en el medio, resultando en el frente de la grieta una concentración de azufre que determinará la velocidad de propagación de la misma ${ }^{[22-25]}$.

Considerando esta serie de premisas y comparando las velocidades de crecimiento obtenidas para las probetas D1, D2 y D3 con una recopilación de datos efectuada por Ford et al. ${ }^{[26]}$ los valores de este trabajo son similares a los de probetas ensayadas a carga constante con descargas periódicas indicadas en dicha recopilación.

Por otra parte, comparando los resultados experimentales con los modelos teóricos de Ford et al. ${ }^{[22,23]}$ para aceros ferríticos de baja aleación (Fig. 8 ), puede verse que los puntos correspondientes a las probetas D1, D2 y D3 están situados dentro de los campos teóricos de crecimiento, lo que significa que dichas probetas han experimentado propagación de grietas acorde con el modelo propuesto para estos materiales, teniendo en cuenta el bajo contenido de azufre del acero SA533B-1 ensayado. Sin embargo, la probeta $\mathrm{D} 8$, con una velocidad de crecimiento del orden de $8,1 \cdot 10^{-8} \mathrm{~mm} / \mathrm{s}$, se sitúa por debajo de la gráfica del modelo teórico para bajos contenidos de azufre, aunque coincide con algunos valores experimentales de la bibliografía ${ }^{[26]}$ en los que se relacionan estos bajos crecimientos con la existencia de picaduras o con plastificaciones en el fondo de la grieta. En el caso de la probeta D8, se observó efectivamente la presencia de picaduras en el fondo de la grieta por ambas superficies externas.

En el resto de las probetas (D4, D5 y D7), el análisis de sus velocidades de crecimiento es más complicada debido a que la propagación ha tenido lugar en más de una zona de la soldadura. La propagación de grieta en la zona de interfase entre el acero ferrítico y el material de soldadura (I-82) se ha producido únicamente en la probeta $\mathrm{D} 4$, con

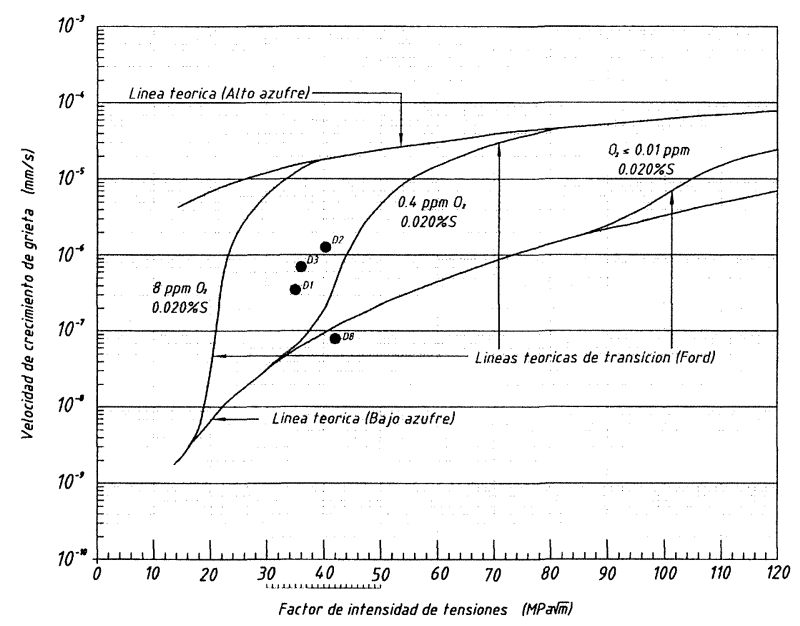

Figura 8. Correlaciones teóricas entre la velocidad de crecimiento de grietas y el factor de intensidad de tensiones (K) acorde con Ford et al.

Figure 8. Theoretically based correlations between crack growth rate and stress intensity factor according to Ford et al. 
un valor de $1,4 \cdot 10^{-6} \mathrm{~mm} / \mathrm{s}$ durante $460 \mathrm{~h}$. Sin embargo, a partir de ese momento creció en algunas zonas en el I-82 y en otras en el SA533B-1 con un valor numérico de crecimiento igual al obtenido en la línea de fusión. Este valor es, a su vez, similar al obtenido para la probeta D2 que experimentó crecimiento en el acero ferrítico y en la primera parte del ensayo de la D7, cuya propagación tuvo lugar en el I-82 y en el SA533B-1.

El dato obtenido en la línea de fusión de la probeta D4 es un resultado a destacar, ya que no existen valores numéricos recogidos en la bibliografía sobre velocidad de crecimiento de grietas en la línea de fusión formada por el acero SA533B-1 y el I-82. El crecimiento mixto experimentado en la probeta D4 en zonas de material ferrítico y de material de soldadura, también se produjo en las probetas D5 y D7. Este comportamiento se puede atribuir a la geometria ondulada de la interfase de soldadura, lo que produce que el plano en que se propaga la grieta se encuentre con los dos materiales en diferentes zonas del espesor de la probeta ${ }^{[27]}$.

Hay pocos datos en la bibliografía sobre materiales de soldaduras y condiciones análogas de ensayo a los aquí expuestos. Haciendo un análisis de cada una de las condiciones que pueden influir en los resultados puede decirse que en un medio con sulfatos esta aleación (I-82), y en general las aleciones base niquel, presentan una mayor susceptibilidad y velocidad de crecimiento de grietas que en medios sin sulfatos ${ }^{[28,29]}$.

Estudios realizados sobre el efecto del oxígeno y la conductividad en la velocidad de crecimiento de grietas en aleaciones base níquel similares al I-82 aunque con menor contenido en cromo, como por ejemplo la aleación I-182, demuestran que un aumento del contenido de oxígeno de 200 a 7.000 ppb supone un aumento de la velocidad de crecimiento en un factor entre 7 y 10 , pudiendo ser este factor, incluso más elevado en el caso de tener una conductividad de $1 \mu \mathrm{S} / \mathrm{cm}$. Sin embargo, se produce un efecto de saturación, ya que ese aumento de la velocidad de crecimiento es menor cuanto mayor es el factor de intensidad de tensiones ${ }^{[30]}$.

Por el contrario, en nuestros ensayos tenemos un factor que contrarresta en parte el aumento de la velocidad, debido a los sulfatos y a los altos niveles de oxígeno y conductividad. Este factor es el hecho de que la propagación de la grieta sea perpendicular a la dirección de las dendritas del I-82, lo que hace disminuir la velocidad de crecimiento de grietas en un orden de magintud con respecto a la propagación en sentido paralelo a las mismas ${ }^{[31]}$.
Para evaluar el crecimiento en el I-82 hay que tener en consideración, además, que la propagación en este material siempre se produce conjuntamente con el avance de la grieta en el material ferrítico (Tabla III), lo que significa que no se han obtenido valores de crecimiento exclusivamente en el material de soldadura.

En un trabajo de Jenssen ${ }^{[32]}$ realizado en el I-82, que fue ensayado en condiciones de química normal de los reactores BWR (200 ppb $\mathrm{O}_{2}$, y baja conductividad) y un factor de intensidad de tensiones comprendido entre 20 y $40 \mathrm{MPa} \sqrt{\mathrm{m}}$, las velocidades obtenidas variaban desde $10^{-9} \mathrm{~mm} / \mathrm{s}$ hasta $5 \cdot 10^{-8} \mathrm{~mm} / \mathrm{s}$. Tomando como referencia estos datos y teniendo en cuenta las condiciones particulares de nuestros ensayos junto con su grado de influencia en la velocidad de crecimiento, expuesto en párrafos anteriores, cabe esperar que la velocidad de propagación de grieta experimentada en el I-82 sea del orden de $10^{-6} \mathrm{~mm} / \mathrm{s}$ o muy próxima. Estos valores, a su vez, son similares a los que se obtienen para el I-82 en un medio con $200 \mathrm{ppb}$ de sulfatos y $200 \mathrm{ppb}$ de $\mathrm{O}_{2}{ }^{[28]}$.

El método de caída de potencial utilizado en las probetas D4, D5 y D7 para la medida de velocidades de propagación de grieta no proporcionó los resultados que cabía esperar, debido a que la sensibilidad de esta técnica para la detección de un aumento de longitud de grieta es dependiente de la homogeneidad del frente de la misma y de la existencia de ligamentos resistentes que van quedando pese al avance de la fisura ${ }^{[33]}$. Avances de grieta en zonas muy localizadas, en materiales diferentes, o que se produzcan de forma interdendrítica, tal como ha ocurrido en dichas probetas, afectan desfavorablemente a la correcta medida de la misma. No obstante, sí fue útil para detectar los momentos de cambio de zonas de propagación y de cambios en la carga.

Se puede realizar un segundo análisis teniendo en cuenta la zona de la soldadura en su conjunto, donde las grietas pueden crecer y propagarse en la interfase y en cualquiera de los materiales que forman la unión soldada. El hecho de que las velocidades de propagación obtenidas sean del mismo orden de magnitud en todas las probetas, independientemente del material en que se han propagado, induce a plantear si es correcto considerar estrictamente la interfase como la línea de fusión, o si por el contrario es más realista considerar esa zona como un conjunto en el que entran a formar parte tanto la interfase como el acero ferrítico y la aleación base níquel en las proximidades de 
aquella, comportándose ese área como un todo con independencia de en qué material se haya localizado la pregrieta de fatiga.

En el análisis global del proyecto, del que ha formado parte este trabajo, y con la aportación de resultados de ensayos complementarios realizados por otros participantes en dicho proyecto ${ }^{[27]}$, se ha concluido que este último es el mecanismo de comportamiento más probable, y que para las características particulares de la soldadura y condiciones de ensayo, las velocidades de propagación no son sensibles a la posición de la pregrieta siempre que se encuentre en las proximidades de la interfase. Sin embargo, no puede generalizarse este comportamiento a otras soldaduras, materiales y medios de ensayo diferentes a los aquí descritos.

\section{CONCLUSIONES}

Después de ensayar siete probetas 1TCT en las peores condiciones de conductividad y contenido de oxígeno que puntualmente pueden presentarse en los reactores tipo BWR en un intervalo de valores del factor de intensidad de tensiones comprendido entre 35 y $56 \mathrm{MPa} \sqrt{\mathrm{m}}$, con el objetivo de obtener velocidad de crecimiento de grietas en la interfase formada por el acero ferrítico SA533B-1y el I-82, se puede concluir:

- Se han obtenido valores medios de velocidad de crecimiento comprendidos entre $8 \cdot 10^{-8} \mathrm{y}$ $2,4 \cdot 10^{-6} \mathrm{~mm} / \mathrm{s}$. Los datos de velocidad de crecimiento de grieta no han mostrado tener una gran dependencia de la posición de la pregrieta. Los valores más bajos de crecimiento se han obtenido en aquellas probetas en las que la pregrieta de fatiga estaba situada muy próxima a la interfase. La longitud de grieta varió significativamente de unas probetas a otras y no se ha encontrando una clara relación entre velocidad y tiempos de ensayo,

- Debido a la superficie ondulada de la interfase de soldadura es difícil hacer coincidir exactamente el frente de grieta con la línea de fusión.

- La posición de la pregrieta influye en la zona de propagación posterior. En los casos en los que se encuentra en el acero ferrítico, la tendencia es a que propague dentro de este mismo material. Por el contrario, cuando la pregrieta está situada en el I-82 se dirige preferentemente hacia la línea de fusión, con crecimiento en algún caso en dicha zona, seguido posteriormente de un avance de la misma en áreas de acero fe- rrítico y de material de soldadura cerca de la interfase. En las probetas con estas características se observaron, además, grietas interdendríticas en el I-82, perpendiculares al plano de crecimiento de la grieta

- Para las condiciones particulares de materiales y ambiente ensayados, la velocidad de propagación de grietas es poco sensible a la situación de las mismas y al material en el que se produzca esa propagación, siempre que se encuentre en las proximidades de la interfase. En este caso, puede considerarse la interfase un área que comprende ambos materiales y la línea de fusión. Sin embargo, no puede generalizarse este comportamiento a otras soldaduras, materiales y medios de ensayo diferentes a los aquí descritos.

\section{Agradecimientos}

Este trabajo ha sido financiado en parte por la Comunidad Económica Europea (F14S-CT95-0018). Los autores agradecen su colaboración a todo el personal del Proyecto de Materiales Estructurales del CIEMAT, en especial a Jose Salido por la realización de los ensayos de corrosión bajo tensión, y a Antonio del Río por los trabajos de microscopía realizados.

\section{REFERENCIAS}

[1] C.D. LUNDIN, Weld. J. Weld. Res. Suppl. 61 (1982) 58-63.

[2] B.T. Timofeev y V.A. Fedorova, Int. J. Pressure Vessel Piping. 64 (1995) 25-42.

[3] C.L. BRIANT y E.L. HALL, Corrosion 43 (1987) 539-548.

[4] A. McMINN, Dissimilar Metal Welds-A Review, Commercial Experience with Dissimilar Metals. Failure Analysis Associates report FaAA-DC-R 90-04-01, WDC17483, Vol.1, 1990.

[5] R.W. WeEKS, Proc. Conf. Int. Symp. on Environmental Degradation of Materials in Nuclear Power SystemsWater Reactors, Mytle Beach, South California, EE.UU., 1983, NACE 1984, pp. 69-86.

[6] A. McMinN, Dissimilar Metal Welds-A Review, Metallurgical and Environmental Effects, Failure Analysis Associates report FaAA-DC-R 90-04-01, WDC17483, Vol. 2, 1990.

[7] A. McMINN, Corrosion 42 (1986) 682-689.

[8] A. McMINN, Proc. Conf. 2th Int. Symp. on Environmental Degradation of Materials in Nuclear Power Systems-Water Reactors, American Nuclear Society, La Grange Park, Illinois, EE.UU., 1986, pp. 108-116. 
[9] A. McMinn y R.A. PAGe, Corrosion 44 (1988) 239-247.

[10] A. McMinn y J.L. Nelson, Proc. Conf. 3th Int. Symp. on Environmental Degradation of Materials in Nuclear Power Systems-Water Reactors, The Metallurgical Society, Warrendale, Pennsylvania, EE.UU., 1988, pp. 389-396.

[11] R.A. Page y A., McMinn, Metall. Trans. 17A (1986) 877-880.

[12] R.A. PAGE, Corrosion 39 (1983) 409-421.

[13] Section 4 from Failure Analysis Associates report, FaAA-LN-R91-10-01, Ln 00007, 1991.

[14] D.D. Macdonald, H. SONG, K. Makela y K. Yoshida, Corrosion 49 (1993) 8-16.

[15] P.L. ANDRESEN, Corrosion 47 (1991) 917-939.

[16] P.L. ANDRESEN, Corrosion 49 (1993) 714-725.

[17] M.D. GómEZ-BRICEÑo et al. Informe interno CIEMAT. ITN/ME-17/DP-93, 1993.

[18] M.P. FeRnÁNDEZ y J. LAPEÑA, Informe interno CIEMAT. ITN/ME-15/SP-96.

[19] ASTM E-399: Test Method for Plane-Strain Fracture Toughness of Metallic Materials, Annual Book of ASTM Standards, ASTM, vol. 03.01, 1996, pp. 407-437.

[20] M. Navas y M.D. Gómez, Nud. Engng. Design, 168 (1987) 183-189.

[21] P. LIDAR, Proc. Conf. 12th Scandinavian Corrosion Congress. Eurocorr '92, 1992, pp. 147-153.

[22] F.P. Ford y P.L. ANdresen, Proc. Conf. Corrosion'89, NACE, Houston, Texas (EE.UU.), 1989, pp. 1-19.

[23] F.P. FORD y P.L. ANDRESEN, Proc. 3rd. Int. Atomic Energy Agency Specialist' Meeting on Subcritical Crack Growth., NUREG/CP-0112, 1990, pp. 105-124.
[24] P. Combrade, M. Foucault y G. Slama, Proc. Conf. 3rd Int. Symp. on Environmental Degradation of Materials in Nuclear Power Systems-Water Reactors, 1987, pp. 269-276.

[25] J.D. AtKInson y J.E. Forrest, Proc. 2nd. Int. Atomic Energy Agency Specialist' Meeting on Subcritical Crack Growth., NUREG/CP-0067, 1990, pp. 105124.

[26] F.P. Ford y P.L. ANdResen, Proc. Conf. 5th Int. Symp. on Environmental Degradation of Materials in Nuclear Power Systems-Water Reactors, Monterey, California, EE.UU., American Nuclear Society Inc., La Grange, Illinois, EE.UU., 1992, pp. 561-570.

[27] D.R. TICE et al. European Commission Euratom Research Framework Programme 1994-1998, Nuclear Fission Safety, Contract F145-CT95-0018, Final Report.

[28] R.A. PAGe y A. MCMINN, Informe EPRI NP-5882M, 1988.

[29] L.G. Lungberg et al., Proc. Conf. 5th Int. Symp. on Environmental Degradation of Materials in Nuclear Power Systems-Water Reactors, Monterey, California, EE.UU., American Nuclear Society Inc., La Grange, Illinois, EE.UU., 1992, pp. 379-384.

[30] P.L. ANDRESEN, Corr. Sci. 44 (1988) 376-385.

[31] L.G. LUNGBERG, Informe EPRI RP 2293-1, Vol.2, 1994.

[32] A. Jenssen et al. Proc. Conf. 7th Int. Symp. on Environmental Degradation of Materials in Nuclear Power Systems-Water Reactors, Breckenridge, Colorado, EE.UU., NACE International, 1995, pp. 563-562.

[33] P.L. ANDRESEN, Informe EPRI TR-107159, 1996. 